\title{
Accurate Evaluation of the Cubic Lattice Green Functions Using Binomial Expansion Theorems
}

\author{
B.A. Mamedov • I.M. Askerov
}

Received: 3 January 2008 / Accepted: 27 March 2008 / Published online: 8 April 2008

(C) The Author(s) 2008. This article is published with open access at Springerlink.com

\begin{abstract}
A new, simple, and efficient technique for the accurate evaluation of the lattice Green functions is presented. Using binomial expansion theorems, these functions are expressed through the binomial coefficients and basic integrals. The extensive test calculations show that the proposed algorithm in this work is the most efficient method in practical computations. Finally, in order to show the practical use of analytical expressions found some computation examples and comparisons with literature are made.
\end{abstract}

Keywords Body centered cubic lattice - Anisotropic face centered cubic lattice $\cdot$ Lattice Green's functions · Binomial coefficients

\section{Introduction}

The evaluation of the lattice Green functions is fundamental to efficient numerical analysis of solid state physics, for example, statistical model of ferromagnetism such as Ising model [1, 2], Heizenberg model [3], spherical model [4], lattice dynamics [5], random walk theory [6], band structure [7], thin films [8]. In the literature, many efficient approaches have been reported for the evaluation of these functions [9-34]. Recently, in [16-25] new mathematical ideas have been introduced, and new algorithm based on the lattice Green functions provides very powerful tools to solve related solid state physics problems. It can be seen from the literature that most of the studies on lattice Green functions are based on elliptic type integral approach. In the literature generally, the formulas for the lattice Green functions have been given in terms of elliptic type integrals and related functions. In the present article we propose that the series expression formulas occur as one infinite sum and in terms of $I_{n}$ basic integral, which make possible the fast and accurate evaluation of the lattice Green functions. This simplification and the use of the computer memory for calculation of binomial coefficients may extend the limits of large arguments to the calculators and result in speedier calculation, should such limits be reached in practice.

B.A. Mamedov ( $\square)$ · I.M. Askerov

Department of Physics, Faculty of Arts and Sciences, Gaziosmanpaşa University, Tokat, Turkey

e-mail: bamamedov@yahoo.com 
In this paper, a new approach to the computation of the lattice Green functions are proposed, which considerably improves its capabilities during numerical evaluations in significant cases. We note that the lattice Green functions have been expressed in terms of binomial coefficients and basic integral functions by using binomial expansion theorems for various range of parameter $w$. However, the series expansions obtained herein give a more accurate and efficient way to compute values for these functions over the entire permissible range of its parameters. Finally, simple examples are presented to compare the effectiveness of the described method with the established formulas for the lattice Green functions in the literature.

\section{Definition and General Expressions for the Lattice Green Functions}

The anisotropic face-centered and simple cubic lattices Green functions are defined as [20], respectively

$$
\begin{aligned}
& G_{1}\left(\alpha_{1}, w_{1}\right)=\frac{1}{\pi^{3}} \int_{0}^{\pi} \int_{0}^{\pi} \int_{0}^{\pi} \frac{d \theta_{1} d \theta_{2} d \theta_{3}}{w_{1}-\left(\cos \theta_{1} \cos \theta_{2}+\cos \theta_{2} \cos \theta_{3}+\alpha_{1} \cos \theta_{3} \cos \theta_{1}\right)} \\
& G_{2}\left(\alpha_{2}, w_{2}\right)=\frac{1}{\pi^{3}} \int_{0}^{\pi} \int_{0}^{\pi} \int_{0}^{\pi} \frac{d \theta_{1} d \theta_{2} d \theta_{3}}{w_{2}-\left(\cos \theta_{1}+\cos \theta_{2}+\alpha_{2} \cos \theta_{3}\right)}
\end{aligned}
$$

where $w_{1}, w_{2}, \alpha_{1}$ and $\alpha_{2}$ are real parameters. In article [18], at the origin for the simple cubic (sc), body-centered cubic (bcc) and face-centered cubic (fcc) lattices a related function of the form

- for simple cubic lattices

$$
G_{1}(w)=\frac{1}{\pi^{3}} \int_{0}^{\pi} \int_{0}^{\pi} \int_{0}^{\pi} \frac{d \theta_{1} d \theta_{2} d \theta_{3}}{w_{1}-\frac{1}{3}\left(\cos \theta_{1}+\cos \theta_{2}+\cos \theta_{3}\right)}
$$

- body-centered cubic lattices

$$
G_{2}(w)=\frac{1}{\pi^{3}} \int_{0}^{\pi} \int_{0}^{\pi} \int_{0}^{\pi} \frac{d \theta_{1} d \theta_{2} d \theta_{3}}{w-\cos \theta_{1} \cos \theta_{2} \cos \theta_{3}}
$$

- face-centered cubic lattices

$$
G_{3}(w)=\frac{1}{\pi^{3}} \int_{0}^{\pi} \int_{0}^{\pi} \int_{0}^{\pi} \frac{d \theta_{1} d \theta_{2} d \theta_{3}}{w-\frac{1}{3}\left(\cos \theta_{1} \cos \theta_{2}+\cos \theta_{2} \cos \theta_{3}+\cos \theta_{3} \cos \theta_{1}\right)}
$$

where $w$ is real parameter.

In order to obtain the expression for lattice Green functions, (1)-(5), we use the following binomial expansion theorems for an arbitrary real or complex $n$ and $|x|>|y|$ (see [35-37]):

$$
(x \pm y)^{n}=\sum_{m=0}^{\infty}( \pm 1)^{m} F_{m}(n) x^{n-m} y^{m}
$$

where $F_{0}(n)=1$ and

$$
F_{m}(n)= \begin{cases}n ! /[m !(n-m) !] & \text { for integer } n \\ \frac{(-1)^{m} \Gamma(m-n)}{m ! \Gamma(-n)} & \text { for noninteger } n\end{cases}
$$


We notice that for $m<0$ the binomial coefficient $F_{m}(n)$ in (7) is zero and the positive integer $n$ terms with negative factorials do not contribute to the summation. The quantities $\Gamma(\sigma)$ in (7) are well known Gamma functions defined by [36, 38]

$$
\Gamma(\sigma)=\int_{0}^{\infty} t^{\sigma-1} e^{-t} d t
$$

Now we can move on to the evaluation of the lattice Green functions. Taking into account (6) in (1)-(5) we obtain for lattice Green functions the following relations, respectively

- for $G_{1}\left(\alpha_{1}, w_{1}\right)$ function

$$
G_{1}\left(\alpha_{1}, w_{1}\right)=\frac{1}{\pi^{3}} \lim _{N \rightarrow \infty} \sum_{i=0}^{N} w_{1}^{-1-i} \sum_{j=0}^{i} \sum_{k=0}^{j} F_{j}(i) F_{k}(j) \alpha_{1}^{k} I_{i-j+k} I_{i-k} I_{j} \quad \text { for } w_{1} \geq \alpha_{1}+2
$$

- for $G_{2}\left(\alpha_{2}, w_{2}\right)$ function

$$
G_{2}\left(\alpha_{2}, w_{2}\right)=\frac{1}{\pi^{3}} \lim _{L \rightarrow \infty} \sum_{i=0}^{L} w_{2}^{-1-i} \sum_{j=0}^{i} \sum_{k=0}^{j} F_{j}(i) F_{k}(j) \alpha_{2}^{k} I_{i-j} I_{j-k} I_{k} \quad \text { for } w_{2} \geq \alpha_{2}+2
$$

- for $G_{1}(w)$ function

$$
G_{1}(w)=\frac{1}{\pi^{3}} \lim _{M \rightarrow \infty} \sum_{i=0}^{M} \frac{1}{(3 w)^{i} w} \sum_{j=0}^{i} \sum_{k=0}^{j} F_{j}(i) F_{k}(j) I_{i-j} I_{j-k} I_{k} \quad \text { for } w \geq 1
$$

- for $G_{2}(w)$ function

$$
G_{2}(w)=\frac{1}{\pi^{3}} \lim _{N^{\prime} \rightarrow \infty} \sum_{i=0}^{N^{\prime}} \frac{\left(I_{i}\right)^{3}}{w^{i+1}} \quad \text { for } w \geq 1
$$

- for $G_{3}(w)$ function

$$
G_{3}(w)=\frac{1}{\pi^{3}} \lim _{L^{\prime} \rightarrow \infty} \sum_{i=0}^{L^{\prime}} \frac{1}{(3 w)^{i} w} \sum_{j=0}^{i} \sum_{k=0}^{j} F_{j}(i) F_{k}(j) I_{i-j+k} I_{i-k} I_{j} \quad \text { for } w \geq 1
$$

In (9)-(13) the indexes $N, L, M, N^{\prime}$ and $L^{\prime}$ are the upper limits of summations, respectively. The quantities $I_{n}$ occurring in (9)-(13) are determined by the relation

$$
I_{n}=\int_{0}^{\pi} \cos ^{n} \varphi d \varphi
$$

In order to evaluate the integral $I_{n}$ we use the formula [36, 39]

$$
I_{n}= \begin{cases}0, & \text { if } n \text { odd } \\ \sqrt{\pi} \frac{\Gamma\left(\frac{n+1}{2}\right)}{\Gamma\left(\frac{n}{2}+1\right)}, & \text { if } n \text { even }\end{cases}
$$




\section{Numerical Results and Discussion}

The paper has presented an efficient and reliably accurate scheme for the direct evaluation of lattice Green functions. The use of a simple numerical computation tool for modeling and simulation can be beneficial in the applications. To demonstrate the accuracy and efficiency of the methods described above we present several numerical results. On the basis of formulas obtained in this paper we constructed a program for computation of the lattice Green functions using Mathematica 5.0 international mathematical software. One can determine the accuracy of computer results obtained from the series expansion formulae by the use of well-known series and analytical formulae in the literature [18-20,26]. The examples of computer calculation for the lattice Green functions (9)-(13) are shown in Tables 1, 2, 3, 4, 5 and 6 . As can be seen from tables, the calculation results of lattice Green functions show

Table 1 The comparative values of lattice Green function $G_{1}\left(\alpha_{1}, w_{1}\right)$ for $N=100$

\begin{tabular}{rlll}
\hline$\alpha_{1}$ & $w_{1}$ & Equation (9) & Equation (2.19) in [20] \\
\hline 1 & 4 & 0.2694162338676949 & 0.2694162338676949 \\
1.5 & 4.8 & 0.2235356974650923 & 0.2235356974650924 \\
3.6 & 8.2 & 0.1311149080401095 & 0.1311149080401095 \\
8.8 & 15.1 & 0.07393384726851592 & 0.07393384726851592 \\
12.1 & 22.4 & 0.04874100147313111 & 0.04874100147313111 \\
32.4 & 52.2 & 0.02157370800729279 & 0.02157370800729277 \\
64.5 & 87.2 & 0.0138515713826008 & 0.0138515713826008 \\
\hline
\end{tabular}

Table 2 The comparative values of lattice Green function $G_{2}\left(\alpha_{2}, w_{2}\right)$ for $L=100$

\begin{tabular}{rrll}
\hline$\alpha_{2}$ & \multicolumn{1}{c}{$w_{2}$} & Equation (10) & Equation (34) in [26] \\
\hline 1.5 & 5 & 0.2219662370202358 & 0.2219662370202359 \\
4.1 & 8.5 & 0.1381536869454573 & 0.1381536869454574 \\
9.3 & 15.4 & 0.0825198994943744 & 0.0825198994943744 \\
19.3 & 25.6 & 0.06010979004222174 & 0.06010979004222174 \\
31.9 & 52.5 & 0.02400854072769508 & 0.02400854072769508 \\
43.1 & 75.2 & 0.0162350508674676 & 0.0162350508674676 \\
84.3 & 117.5 & 0.01222189500170813 & 0.01222189500170813 \\
\hline
\end{tabular}

Table 3 The comparative values of lattice Green function $G_{1}(w)$ for $M=100$

\begin{tabular}{rll}
\hline$w$ & Equation (11) & Equation (4.13) in [19] \\
\hline 2 & 0.523378692134745 & 0.523378692134745 \\
3.8 & 0.2662865641363358 & 0.2662865641363358 \\
24.3 & 0.04116388686510464 & 0.04116388686510463 \\
41.3 & 0.02421544155071478 & 0.02421544155071479 \\
54.8 & 0.01824918808427292 & 0.01824918808427292 \\
74.3 & 0.01345935656575595 & 0.01345935656575595 \\
174.3 & 0.005737266127665814 & 0.005737266127665817 \\
\hline
\end{tabular}


Table 4 The comparative values of lattice Green function $G_{2}(w)$ for $N^{\prime}=100$

\begin{tabular}{rll}
\hline$w$ & Equation (12) & Equation (3.3) in [18] \\
\hline 1.8 & 0.5804119188514366 & 0.5804119188514366 \\
13.6 & 0.07357921824417764 & 0.07357921824417764 \\
33.6 & 0.0297652012728565 & 0.0297652012728565 \\
45.6 & 0.02193114313268718 & 0.02193114313268718 \\
85.6 & 0.01168244229360323 & 0.01168244229360323 \\
145.5 & 0.00687289281532574 & 0.00687289281532574 \\
225.5 & 0.004434600701635175 & 0.004434600701635175 \\
\hline
\end{tabular}

Table 5 The comparative values of lattice Green function $G_{3}(w)$ for $L^{\prime}=100$

\begin{tabular}{rll}
\hline$w$ & Equation (13) & Equation (4.2) in [18] \\
\hline 1.4 & 0.7623279875448653 & 0.7623279875448653 \\
5.8 & 0.1728699424811432 & 0.1728699424811432 \\
25.8 & 0.03876460738828515 & 0.03876460738828516 \\
41.5 & 0.02409756105746882 & 0.02409756105746883 \\
81.5 & 0.01227009322524353 & 0.01227009322524353 \\
174.4 & 0.005733960694430566 & 0.005733960694430564 \\
381.1 & 0.002603490146674031 & 0.002603490146674031 \\
\hline
\end{tabular}

Table 6 Convergence of derived expression for $G_{3}(w)$ as a function of summation limits $L^{\prime}$

\begin{tabular}{lll}
\hline$L^{\prime}$ & $w=18.1$ & $w=24.5$ \\
\hline 10 & 0.05526294497056553 & 0.04082207323315965 \\
15 & 0.05526294497056554 & 0.04082207323315965 \\
20 & 0.05526294497056554 & 0.04082207323315965 \\
25 & 0.05526294497056554 & \\
\hline
\end{tabular}

good rate of convergence with literature under range of parameters $[16,18,19,26]$. Tables 6 and 7 show that the convergence properties of the two expressions (9)-(10) considered vary widely. As can be seen from Tables 6 and 7, (9) and (10) display the most rapid convergence to the numerical result, with seventeen digits stable and correct by the fiftieth terms in the infinite summation.

The comparative computer time required for the calculation the lattice Green functions are not given in the tables due to the fact that the comparison cannot be made with the different computers used in the literature. It is seen from the algorithm presented for lattice Green functions that our CPU times are satisfactory. For instance, using (10) and (4.26) in [20] for $G_{2}(8.3,17.4)$, CPU times takes about $0.05 \mathrm{~ms}$ and $0.07 \mathrm{~ms}$, respectively. In conclusion, by means of a binomial expansion theorem, we have obtained the new simple accurate general expressions of the lattice Green functions. 
Table 7 Convergence of derived expression for $G_{1}\left(\alpha_{1}, w_{1}\right)$ as a function of summation limits $N$

\begin{tabular}{rll}
\hline$N$ & $\alpha_{1}=24.4 ; w_{1}=30.8$ & $\alpha_{1}=15.2 ; w_{1}=42.3$ \\
\hline 20 & 0.04132414783034567 & 0.02447839469388765 \\
30 & 0.04135511951316856 & 0.02447839469474832 \\
40 & 0.04135923109073288 & 0.02447839469474839 \\
50 & 0.0413598555600943 & 0.02447839469474839 \\
60 & 0.04135995800446002 & \\
90 & 0.04135997942362581 & \\
120 & 0.04135997955759622 & \\
150 & 0.04135997955857419 & \\
180 & 0.04135997955858199 & \\
190 & 0.04135997955858204 & \\
200 & 0.04135997955858204 & \\
\hline
\end{tabular}

Open Access This article is distributed under the terms of the Creative Commons Attribution Noncommercial License which permits any noncommercial use, distribution, and reproduction in any medium, provided the original author(s) and source are credited.

\section{References}

1. Economou, E.N.: Green Function in Quantum Physics, 2nd edn. Springer, Berlin (1983)

2. Brout, R.: Statistical mechanical theory of ferromagnetism. High density behavior. Phys. Rev. 118, 10091019 (1960)

3. Dalton, N.W., Wood, D.W.: Critical behaviour of the simple anisotropic Heisenberg model. Proc. Phys. Soc. (Lond.) 90, 459-475 (1967)

4. Lax, M.: Molecular field in the spherical model. Phys. Rev. 97, 629-640 (1955)

5. Kato, N.: A foundation for the statistical dynamical theory of diffraction. Acta Crystallogr. A 47, 1-11 (1991)

6. Montroll, E.W.: Random walks in multidimensional spaces, especially on periodic lattices. SIAM J. Appl. Math. 4, 241-260 (1956)

7. Koster, G.F., Slater, D.C.: Simplified impurity calculation. Phys. Rev. 96, 1208-1223 (1954)

8. Wachniewski, A.: Lattice Green's function for thin films. The S.C.(001) thin film Green's function. Phys. Status Solidi B 104, 555-562 (1981)

9. Li, Q., Soukoulis, C., Economou, E.N., Grest, G.S.: Anisotropic tight-binding model for localization. Phys. Rev. B 40, 2825-2830 (1989)

10. Heiner, E., Schneider, J.: A polynomial approximation for the imaginary part of the lattice Green's function for the simple cubic lattice. Phys. Status Solidi B 49, K37-K39 (1972)

11. Watson, G.N.: Three triple integrals. Q. J. Math. 10, 266-276 (1939)

12. Inoue, M.: Lattice Green's function for the body-centered cubic lattice. J. Math. Phys. 16, 809-812 (1975)

13. Joyce, G.S.: Lattice Green function for the anisotropic face centred cubic lattice. J. Phys. C: Solid State Phys. 4, L53-L56 (1971)

14. Joyce, G.S.: Exact evaluation of the body centred cubic lattice Green function. J. Phys. C: Solid State Phys. 4, 1510-1512 (1971)

15. Joyce, G.S.: On the simple cubic lattice Green function. Philos. Trans. R. Soc. A 273, 583-610 (1973)

16. Joyce, G.S.: On the cubic lattice Green functions. Proc. R. Soc. A 445, 463-467 (1994)

17. Glasser, M.L.: Closed form expressions for a class of lattice Green functions. J. Phys.: Conf. Ser. 42, 95-97 (2006)

18. Joyce, G.S.: Singular behaviour of the cubic lattice Green functions and associated integrals. J. Phys. A: Math. Gen. 34, 3831-3839 (2001)

19. Joyce, G.S.: On the cubic modular transformation and the cubic lattice Green functions. J. Phys. A: Math. Gen. 31, 5105-5115 (1998) 
20. Joyce, G.S., Delves, R.T., Zucker, I.J.: Exact evaluation of the Green functions for the anisotropic facecentred and simple cubic lattices. J. Phys. A: Math. Gen. 36, 8661-8672 (2003)

21. Joyce, G.S.: Evaluation of the Watson integral and associated logarithmic integral for the $d$-dimensional hypercubic lattice. J. Phys. A: Math. Gen. 34, 7349-7354 (2001)

22. Joyce, G.S.: Exact evaluation of the simple cubic lattice Green function for a general lattice point. J. Phys. A: Math. Gen. 35, 9811-9828 (2002)

23. Joyce, G.S., Delves, R.T.: The product forms for the simple cubic lattice Green function II. J. Phys. A: Math. Gen. 37, 5417-5447 (2004)

24. Joyce, G.S., Delves, R.T.: The product forms for the simple cubic lattice Green function I. J. Phys. A: Math. Gen. 37, 3645-3671 (2004)

25. Guseinov, I.I., Mamedov, B.A.: A Unified treatment of the lattice Green function, generalized Watson integral and associated logarithmic integral for the $d$-dimensional hypercubic lattice. Philos. Mag. 87, 1107 (2007)

26. Delves, R.T., Joyce, G.S.: On the Green function for the anisotropic simple cubic lattice. Ann. Phys. 291, 71-133 (2001)

27. Delves, R.T., Joyce, G.S.: Exact evaluation of the Green function for the anisotropic simple cubic lattice. J. Phys. A: Math. Gen. 34, L59-L65 (2001)

28. Katsura, S., Horiguchi, T.: Lattice Green's function for the body-centered cubic lattice. J. Math. Phys. 12, 230-231 (1971)

29. Mano, K.: Remarks on the Green's functions for the cubic lattices. J. Math. Phys. 16, 1726-1728 (1975)

30. Morita, T.: Useful procedure for computing the lattice Green's function-square, tetragonal, and bcc lattices. J. Math. Phys. 12, 1744-1747 (1971)

31. Morita, T., Horiguci, T.: Calculation of the lattice Green's function for the bcc, fcc, and rectangular lattices. J. Math. Phys. 12, 986-992 (1971)

32. Sakaji, A., Hijjawi, R.S., Shawagfeh, N., Khalifeh, J.M.: Int. J. Theor. Phys. 41, 973 (2002)

33. Sakaji, A., Hijjawi, R.S., Shawagfeh, N., Khalifeh, J.M.: Lattice Green's function for the body-centered cubic lattice. Int. J. Theor. Phys. 41, 973-983 (2002)

34. Glasser, M.L., Boersma, J.: Exact values for the cubic lattice Green functions. J. Phys. A: Math. Gen. 33, 5017-5023 (2000)

35. Gradshteyn, I.S., Ryzhik, I.M.: Tables of Integrals, Sums, Series and Product, 4th edn. Academic Press, San Diego (1980)

36. Abramowitz, M., Stegun, I.: Handbook of Mathematical Functions. Dover, New York (1972)

37. Guseinov, I.I., Mamedov, B.A.: Evaluation of overlap integrals with integer and noninteger $n$ Slater-type orbitals using auxiliary functions. J. Mol. Mod. 8, 272-276 (2002)

38. Guseinov, I.I., Mamedov, B.A.: Evaluation of incomplete gamma functions using downward recursion and analytical relations. J. Math. Chem. 36, 341-346 (2004)

39. Mamedov, B.A., Tapramaz, R., Merdan, Z.: Calculation of generalized elliptic type integrals using the binomial expansion theorem. Appl. Math. Comput. 168, 333-341 (2005) 